\title{
Lateral masking vertically and horizontally
}

\author{
LARRY CHAMBERS and GEORGE WOLFORD \\ Dartmouth College, Hanover, New Hampshire
}

\begin{abstract}
The greater effectiveness of peripheral over central masks was investigated in an experiment with 16 separate target-mask displays: a single mask letter above, to the left of, below, or to the right of a single target letter, and the pair of letters above, to the left of, below, and to the right of fixation. Close target-mask spacing was used, and the center points of the target-mask pairs were held constant. Peripheral masking was more effective than foveal masking in both the vertical and horizontal directions. Furthermore, masks on the same radius as the target (whether peripheral or central) were more effective than masks off that radius. The results were consistent with Wolford's (1975) feature-perturbation model of visual masking.
\end{abstract}

Accuracy at detection of a briefly presented target letter is impaired by a mask letter presented simultaneously nearby. This effect has been called lateral interference or masking. Several properties of lateral masking are well established. First, lateral masking is more detrimental in the periphery than in the fovea (Matthews, 1973; Wolford \& Hollingsworth, 1974). Second, it is more detrimental with close target-mask spacing than with wide target-mask spacing (Flom, Weymouth, \& Kahneman, 1963; Wolford \& Chambers, 1983). Third, masks placed on the peripheral sides of target letters are more effective than masks placed on the foveal sides (Banks, Larson, \& Prinzmetal, 1979; Bouma, 1973). This last effect was of special concern in the present experiment.

Two different types of explanations of the masking asymmetry have been proposed, one based on feature perturbations and one based on the center points of target-mask displays. Wolford's (1975) feature-perturbation model proposes that features of letters have a probability of drifting, or being mislocated, in any direction, but mislocations in a foveal direction are more common than those in any other direction. Features of a mask letter that migrate and combine with the features of a target letter can interfere with identification of that target. In the model, because features more often drift foveally than peripherally, features of a peripheral mask will interfere with target identification more often than will features of a foveal mask. Banks et al. (1979) proposed an alternative account based on the observation that the center-point distance of the target-mask configuration from fixation had been confounded in previous work with central/peripheral placement of masks. In particular, the center points of displays with peripheral masks had been farther from the fixation point than the center points of displays with central masks.

The authors' mailing address is: Department of Psychology, Dartmouth College, Hanover, New Hampshire 03755.
Banks et al.'s first two experiments demonstrated that the differences in center-point eccentricities could account for differences in effectiveness between central and peripheral masks.

One obvious way to contrast these two models is to hold center-point distance constant while varying mask location relative to the target. Chastain and Lawson (1979) held center points of target-mask displays constant and still found peripheral masking to be more effective than foveal masking. They used targets and masks to the left or right of the fixation point. The present study extended the research of Chastain and Lawson by including a greater variety of target and mask locations.

\section{METHOD}

Subjects

Eight undergraduates enrolled in an introductory psychology course participated for course credit.

\section{Stimuli and Apparatus}

The target letters $\mathrm{F}, \mathrm{C}, \mathrm{U}$, and $\mathrm{T}$ and the mask letter $\mathrm{H}$ were constructed using dot matrices, $0.3 \mathrm{deg}$ square. Since feature perturbations are assumed to be limited to about $0.5 \mathrm{deg}$ (Wolford, 1975), the target and mask were separated by $0.43 \mathrm{deg}$ vertically or horizontally, center to center. The center point of the target-mask pair was always $5.0 \mathrm{deg}$ from the fixation point (again, vertically or horizontally).

There were 16 possible stimulus displays, all possible combinations of four target-mask display locations relative to the fixation point at the center of the screen and four mask locations relative to the target. Figure 1 illustrates 4 of the possible displays, only 1 of which appeared on a given trial. In the figure, " $T$ " refers to target and " $M$ " to mask. Of particular interest were four peripheral-mask displays: mask above the target with the display above the fixation cross, mask to the left of the target with the display to the left of fixation, mask below the target with the display below fixation, and mask to the right of the target with the display to the right of fixation. Stimuli were presented on video monitors controlled by Terak 8510 minicomputers. The viewing distance of $71 \mathrm{~cm}$ was ensured by a wooden hood with eyepiece attached to the front of the monitor. All letters were shown as black characters on a white screen. 


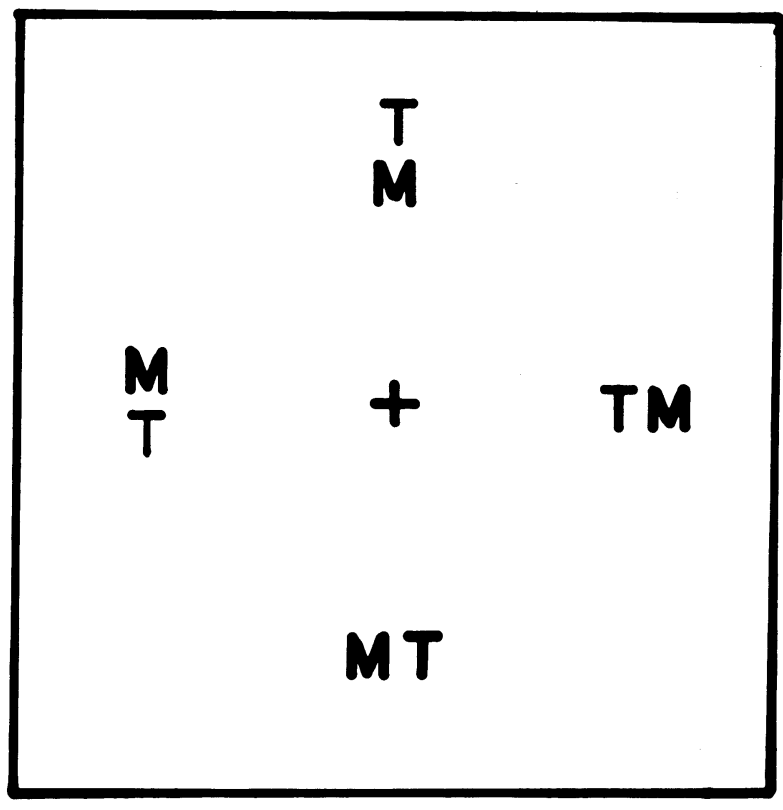

Figure 1. Examples of stimulus configurations using the four mask positions relative to the target and the four target positions relative to the fixation point.

\section{Procedure}

Each trial began with a plus sign centered on the screen, serving as the fixation cross. After $1 \mathrm{sec}$, the target-mask display was shown for $100 \mathrm{msec}$. The subject pressed one of four designated keys on the Terak keyboard to indicate choice of letter and then pressed a separate key to initiate the next trial. The subject was instructed to look at the fixation cross until the target and mask were presented.

There were 64 trials in each block, including each of the 16 displays crossed with each of the four letters. The subject saw 10 blocks of 64 trials. The first 2 blocks served as practice and were not included in the data analysis.

\section{RESULTS AND DISCUSSION}

Table 1 shows mean letter-detection accuracy for each of the 16 displays (collapsed over target letters). Scores along a given row are those for a target position relative to the fixation point; the scores in a given column are for a position of the mask relative to the center point of the target-mask stimulus configuration. Scores along the main diagonal are those of peripheral masks. Three of the four show decrements in percentage

Table 1

Percentage of Correct Detection as a Function of Target Location and Mask Location

\begin{tabular}{lcccc}
\hline \multirow{2}{*}{$\begin{array}{c}\text { Mask } \\
\text { Location }\end{array}$} & Above & Right & Below & Left \\
\cline { 2 - 5 } & 47 & 80 & 70 & 83 \\
Above & 47 & 80 & 79 & 83 \\
Right & 63 & 83 & 60 & 80 \\
Below & 45 & 75 & 66 & 74 \\
Left & 50 & & &
\end{tabular}

correct compared with the mean of the other three displays at their locations, and in the bottom and leftof-fixation-point positions, the mask-peripheral displays were the lowest in accuracy in their column. The planned comparison of the 4 mask-peripheral displays compared with the other 12 was highly reliable $[\mathrm{F}(1,63)$ $=16.13, \mathrm{p}<.0002$ ]. The peripheral mask clearly hindered letter detection relative to the other masks.

Each of the main effects of target letters, position of the display relative to the fixation point, and targetmask display were significant, as were all two-way interactions of these factors. Displays above and below the fixation point showed less overall accuracy than did displays to the left or right. There also was better performance on displays in which the target was to the left of the mask, for example, "TH," than on the righthand targets, for example "HT," in all four locations relative to the fixation point. We subtracted this main effect of mask location from the scores in Table 1. Since this main effect is orthogonal to the interaction depicted in Table 1, this does not affect the nature of the interaction. These derived scores are presented in Table 2 . In the table, the scores along the main diagonal (for peripheral masks) are smaller than any other score in that column for all target locations except left of fixation. These adjusted means more clearly indicate the decrement of peripheral masks relative to other mask locations.

An inspection of Table 2 reveals that, after peripheral masks, central masks were the next most effective masks. Thus, masks on the target/fixation-point radius were more effective than masks that lay off that radius. The superiority of masks on the same radius may have important methodological implications. Some lateralmasking studies use circular displays, whereas others use linear displays. The current research suggests that the effect of lateral masking may not be equivalent in the two cases.

The effectiveness of radial masks is consistent with the perturbation model. In the current paradigm, the subjects were presented with two letters on each trial. The only cues the subjects had as to which was the target on a particular trial were the identities of the two letters. On some trials, it might have aided the subject to identify the mask and eliminate it from further consideration. The same logic that earlier led us to predict the greater effectiveness of peripheral masks

Table 2

Percentage of Correct Detection at Target and Mask Locations With the Main Effect of Mask Location Partialed Out

\begin{tabular}{lcccc}
\hline & \multicolumn{4}{c}{ Target Location } \\
\cline { 2 - 5 } Location & Above & Right & Below & Left \\
\hline Above & 47 & 80 & 70 & 83 \\
Right & 57 & 74 & 73 & 77 \\
Below & 48 & 87 & 63 & 83 \\
Left & 54 & 79 & 70 & 78 \\
\hline
\end{tabular}


leads us to predict that the peripheral target will interfere with the identification of the mask. In this hypothesis, masks and targets that lie on different radii will have the smallest joint interference.

Holding constant the center points of target-mask displays did not eliminate the asymmetry between peripheral and nonperipheral masking. Letter-detection accuracy was lowest with peripheral masks, including peripheral masks above or below the target. These results are consistent with the perturbation model (Wolford, 1975).

\section{REFERENCES}

Banks, W. P., Larson, D. W., \& Prinzmetal, W. Asymmetry of visual interference. Perception \& Psychophysics, 1979, 25, 447-456.
Bouma, H. Visual interference in the parafoveal recognition of initial and final letters of words. Vision Research, 1973, 13, 767-782.

Chastain, G., \& Lawson, L. Identification asymmetry of parafoveal stimulus pairs. Perception \& Psychophysics, 1979, 26, 363-368.

Flom, M. C., Weymouth, F. W., \& Kahneman, D. Visual resolution and contour interaction. Journal of the Optical Society of America, 1963, 53, 1026-1032.

MatThews, M. L. Locus of presentation and the selective masking effect. Canadian Journal of Psychology, 1973, 27, 343-349.

Wolford, G. Perturbation model for letter identification. Psychological Review, 1975, 82, 184-199.

Wolford, G., \& Chambers, L. Lateral masking as a function of spacing. Perception \& Psychophysics, 1983, 33, 129-138.

Wolford, G., \& Hollingsworth, S. Lateral masking in visual information processing. Perception \& Psychophysics, 1974, 16, 315-320.

(Manuscript received for publication August 22, 1983.) 